\title{
Experimental investigation of the effect of insulator sleeve length on the time to pinch and multipinch formation in the plasma focus facility
}

\author{
M. Momenei ${ }^{1} \cdot$ Z. Khodabakhshei $^{1} \cdot$ N. Panahi ${ }^{2} \cdot$ M. A. Mohammadi ${ }^{3}$
}

Received: 7 August 2016/ Accepted: 1 December 2016/Published online: 5 January 2017

(C) The Author(s) 2017. This article is published with open access at Springerlink.com

\begin{abstract}
The length of insulator sleeve is varied to investigate its effect on the pinch formation in the plasma focus facility. In this paper, the effect of insulator length on the time to pinch at various pressures and working voltages in the $1.15 \mathrm{~kJ}$ Mather type plasma focus is investigated. The results show that with $4.5 \mathrm{~cm}$ insulator length the time to pinch at all pressures is minimum. Other results also confirm that with increasing of pressure the time to pinch is increased. Moreover, with increasing working voltage the time to pinch is decreased. Pictures, captured using a digital single lens reflex (DSLR) Canon EOS 7D system, show that multipinch phenomenon is formed.
\end{abstract}

Keywords Plasma focus · Insulator sleeve $\cdot$ Multipinch

\section{Introduction}

Plasma focus device (PFD) is an effective device in laboratory for production of high-temperature $(\sim 1 \mathrm{keV})$ and high-density $\left(\approx 10^{25}-10^{26} \mathrm{~m}^{-3}\right)$ plasma. PFD was developed in the early 1960s in the former Soviet Union (Filippov type) [1] and in the USA (Mather type) [3] independently. The PFD was initially considered as a fast neutron source $[3,4]$. It is also a rich source of soft and hard X-rays [5, 6], highly energetic ions [7, 8] and

M. A. Mohammadi

mohammadidorbash@yahoo.com

Faculty of Physics, University of Shahrood, Shahrood, Iran

2 Department of Physics, Bandar Abbas Branch, Islamic Azad University, Bandar Abbas, Iran

3 Department of Atomic and Molecular Physics, Faculty of Physics, University of Tabriz, Tabriz, Iran relativistic electrons [9]. The X-ray emission from PFD has been used for defectoscopy, X-ray lithography activation of enzymes, micro-machining and radiography [10-13]. The energetic ions have been used for material processing such as ion implantation and thin films [14-16]. PFD has also been used as a pump source for lasers [17]. Plasma produced in PFD can be affected with the plasma focus insulator sleeve length and PFD working voltage and pressure. Recently, experimental studies have been carried out on the effects of insulator sleeve length and pressure on time to pinch and current sheath structure $[18,19]$. The effect of insulator sleeve length on X-ray emission has also been reported by Rawat et al. [20]. In $[21,22]$ Zhang et al. and Zakaullah et al. show that the $\mathrm{X}$-ray and neutron yield is affected by insulator sleeve length. The current sheath formation dynamics and its structure for different insulator lengths in plasma focus device are investigated by Seng et al. [23]. The current sheath dynamics and multipinch phenomena have also been reported by Mohammadi et al. [24].

In this study, we have shown that the change in insulator sleeve length, pressure and working voltage affects the pinch time. The formation of multipinch phenomena is also reported.

\section{Experimental setup}

The present investigation was performed on a simple single capacitor DPF device designated at the Shahrood University (SHUPF). It is a Mathertype focus device, energized by a single $16 \mu \mathrm{F}, 12 \mathrm{kV}$ fast discharging capacitor, with a maximum energy storage of $1.15 \mathrm{~kJ}$. In our investigation, the device was operated at a charging voltage ranging between 7 and $9 \mathrm{kV}$. In our plasma focus, SHUPF, 
cylindrical anode made of copper has $60 \mathrm{~mm}$ in length and $20 \mathrm{~mm}$ in diameter. The cathode was built in six brass rods each of $10 \mathrm{~mm}$ diameter and $60 \mathrm{~mm}$ length and symmetrically located around the anode. The device was evacuated to a vacuum $(\sim 0.005$ Torr $)$ by a rotary pump and was filled with argon gas to a different pressure (1-1.6 Torr) before the operation.

An insulator sleeve of Pyrex glass with fixed $24 \mathrm{~mm}$ outer diameter, in different effective lengths $35,40,45$ and $50 \mathrm{~mm}$, separates anode and cathode, as shown in Fig. 1. For determination of the temporal pinch zone, high-voltage probe is used. All data are captured with the GPS $200 \mathrm{MHz}$ digital oscilloscope. A digital single lens reflex (DSLR) Canon EOS 7D was used for the time-integrated plasma column photography. The open shutter camera with an analyzer and polarizer was fixed at a distance of $15 \mathrm{~cm}$ in front of the window.

\section{Results and discussion}

In Fig. 2 typical signal of voltage probe is shown. The first peak (I) of signal coincides with breakdown phase and the second and third peaks (II, III) are pinch signals. Time distance between I and II (the time from start of discharge to the pinch) is time to pinch. In Fig. 3 variation of time to pinch versus insulator sleeve for different pressure is shown. As seen, the minimum and maximum time to pinch are $4.99 \pm 0.04(\mu \mathrm{s})$ to $4.5 \mathrm{~cm}$ at 1 Torr and $7.36 \pm 0.06$ $(\mu \mathrm{s})$ for $3.5 \mathrm{~cm}$ at 1.4 Torr, respectively. At 1.6 Torr pressure with $3.5 \mathrm{~cm}$ insulator sleeve length pinch is not formed. At all pressure, the time to pinch for insulator with $4.5 \mathrm{~cm}$ is minimum. This result confirms that at all insulators, there is one pressure, which time to pinch is minimum. At all pressures when we deviate from an insulator sleeve with $4.5 \mathrm{~cm}$ length the time to pinch increases. This means that this insulator sleeve length is the optimum length of this plasma focus facility. In Fig. 4 variation of

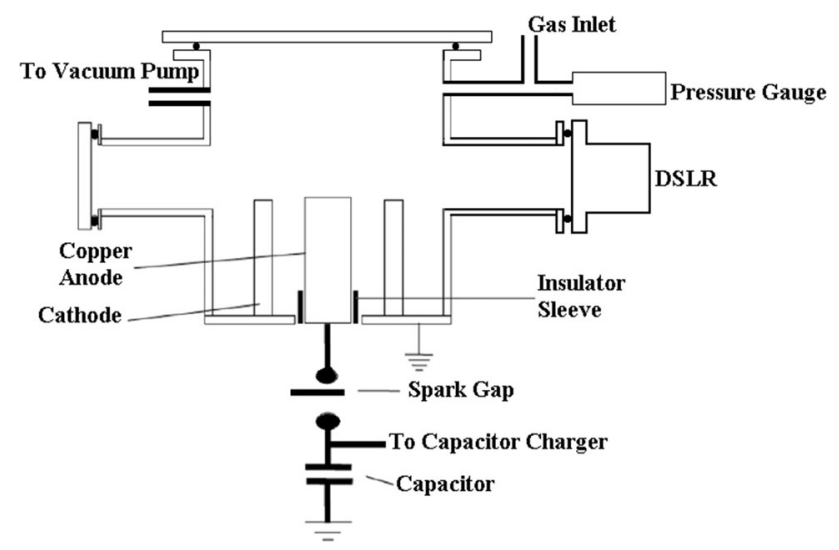

Fig. 1 The schematic view of SHUPF

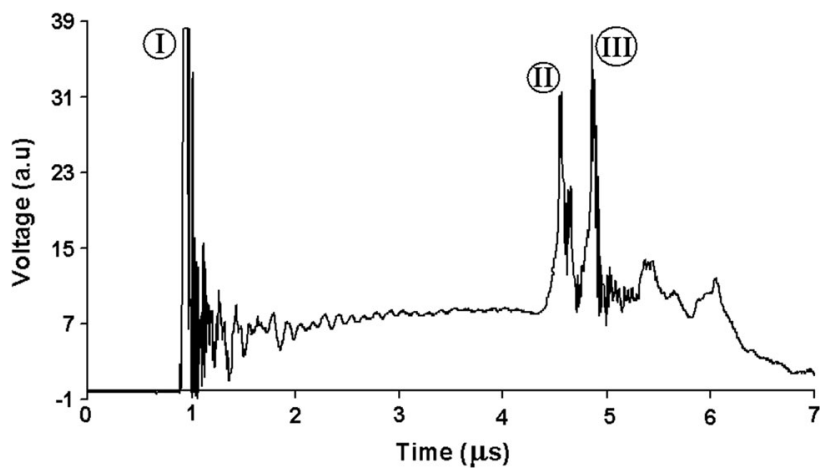

Fig. 2 Typical signal of voltage probe

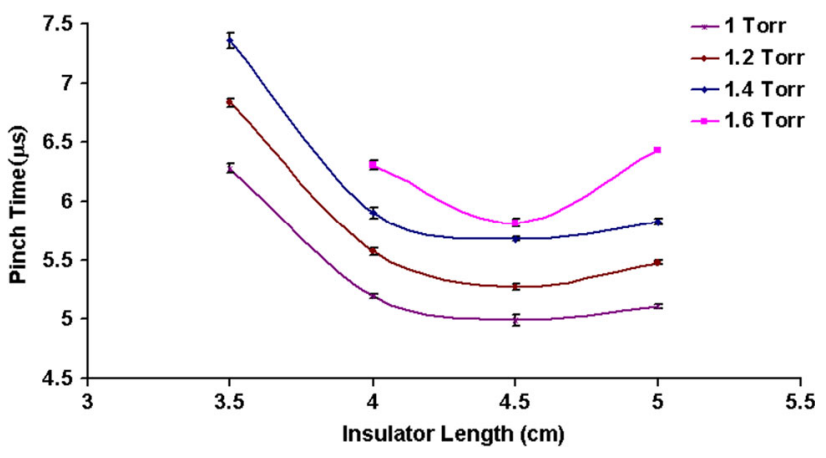

Fig. 3 Variation of time to pinch with insulator sleeve length for different pressure

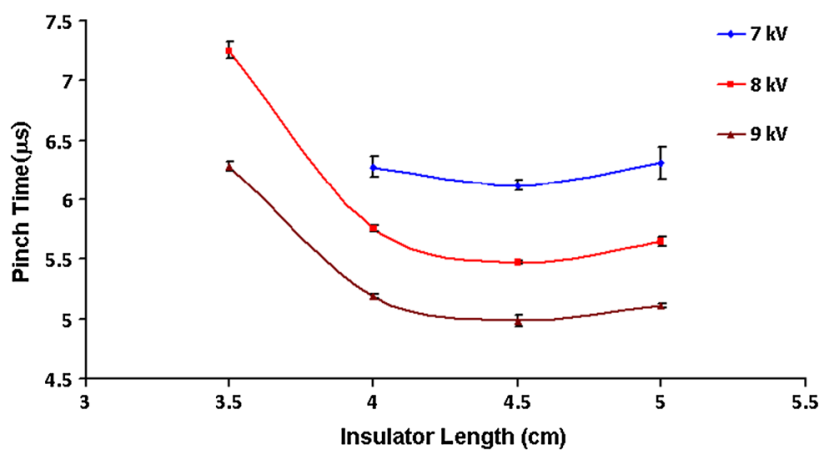

Fig. 4 Variation of time to pinch with insulator sleeve length for different voltage

time to pinch versus insulator sleeve length for different working voltage is shown. This figure shows that with increasing of voltage for all insulator sleeve length time to pinch is decreased. With increasing of voltage the current is increased, and then the Lorentz force for driving current sheath is increased. Also the result of this figure shows that time to pinch at insulator with $4.5 \mathrm{~cm}$ length for all voltages is minimum. This means that the optimum length is independent of working voltage and pressure. When the insulator length is increased or decreased from the optimum value $(4.5 \mathrm{~cm}$ length) the time to pinch is increased. The modification factor is defined as follows [25]: 

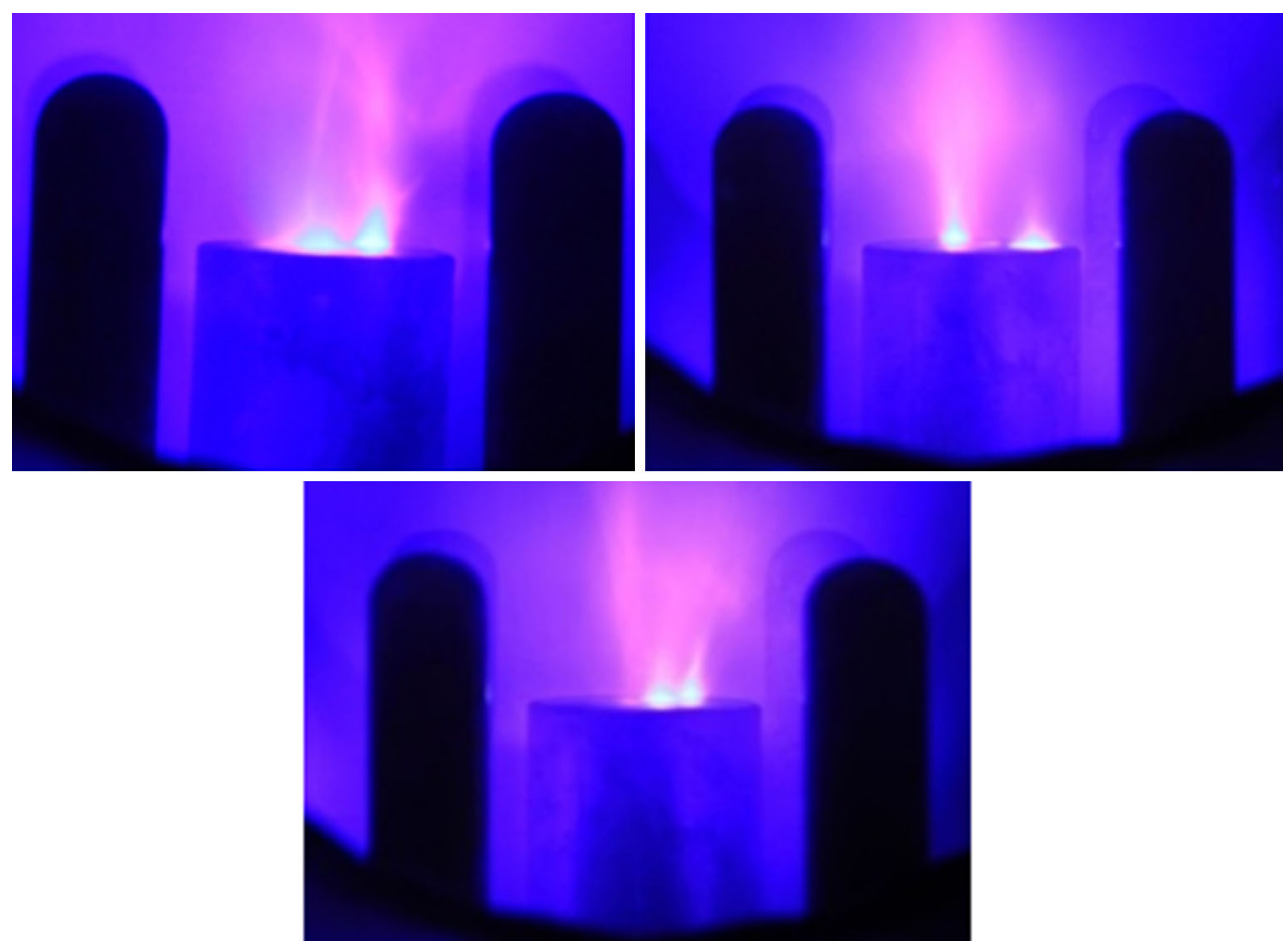

Fig. 5 Time integrate picture of pinch zone

$F=f_{\mathrm{c}} / \sqrt{f_{\mathrm{m}}}$

where $f_{\mathrm{c}}$ and $f_{\mathrm{m}}$ are fraction of current and mass swept factor driving the plasma sheath, respectively. With the deviating of optimum value of insulator sleeve length, the leakage current will be increased and the modification factor is decreased. With considering of modification factor the current sheath velocity equation at axial velocity is defined as [26]

$U_{a}=F\left[\frac{\mu \ln C}{4 \pi^{2}\left(C^{2}-1\right)}\right]^{1 / 2} \frac{I_{0}}{a \sqrt{\rho}}$.

This equation shows that with decreasing the modification factor the axial velocity is decreased, so the time to pinch is increased. The optimum insulator sleeve length corresponds to the conditions for uniform discharge development and its take off across the insulator sleeve surface. When the sleeve is too long the increased inductance may cause the current sheath to remain at the sleeve surface for longer period of time. When the sleeve is too short, the rapid current sheath development may cause spoke formation. As a result, when the insulating sleeve is not of appropriate length, the current sheath no longer remains uniform, and the so-called filaments or spokes are developed [26].

The sheath formation time is given as [27] $t_{\mathrm{f}}=\left(\frac{2 \pi r_{\mathrm{s}} l_{\mathrm{s}} d_{\mathrm{s}} L_{\mathrm{i}}}{\eta} w_{\mathrm{is}}\right)^{1 / 2} \cdot \frac{1}{U}$.

where $U$ is the working voltage, $r_{\mathrm{s}}$ and $l_{\mathrm{s}}$ are the radius and the length of insulator, respectively; $L_{\mathrm{i}}$ is the inductance; $d_{\mathrm{s}}$ is the sheath thickness; $\eta$ is the efficiency of energy fed to the discharge and $w_{\text {is }}$ is the energy density. When the insulator sleeve length is longer than the optimum value, the sheath formation time is increased and then the time to pinch is increased. Equation 3 shows that with increasing of working voltage the sheath formation time decreased, which is confirmed by Fig. 4 .

For the time-integrated study of plasma column a digital single lens reflex (DSLR) Canon EOS 7D is used. Timeintegrated picture of pinch zone is shown in Fig. 5. This figure shows that the multipinch is formed on top of the anode surface. The II and III peaks of the voltage probe signal, which is shown in Fig. 2, also confirm that the multipinch is formed. The multipinch formation can be explained as follows: in the Lee model the mass carried by current sheath at the position $\mathrm{z}$ is [28]

$\rho \pi\left(b^{2}-a^{2}\right) m_{\mathrm{f}} z$.

In this equation $\rho, b$ and $a$ are gas density, cathode radius and anode radius, respectively. $m_{\mathrm{f}}$ is the mass factor being less than one. This equation explains that the current sheath cannot carry $100 \%$ of gas, but some gas is left back 
near the insulator sleeve. After the first pinch/compression phase, indicated by the first peak in the voltage probe signal, shown in Fig. 2, another discharge on the insulator is produced and a second current sheath is produced which, owing to the low density of gas in front of it moves much faster and collapses at the anode top as the second pinch/compression phase.

\section{Conclusion}

Pinch formation time with the various insulator sleeve length is investigated. It was found that at all insulators we have one minimum time to pinch. It was obtained that with insulator with $4.5 \mathrm{~cm}$ length the time to pinch at all pressure is minimum. The average pinch time with different pressure shows that with increasing of pressure the time to pinch increased. Experimentally, it was shown that at a higher voltage the time to pinch is decreased. Experiments demonstrate that the multipinch phenomenon is formed.

Open Access This article is distributed under the terms of the Creative Commons Attribution 4.0 International License (http://crea tivecommons.org/licenses/by/4.0/), which permits unrestricted use, distribution, and reproduction in any medium, provided you give appropriate credit to the original author(s) and the source, provide a link to the Creative Commons license, and indicate if changes were made.

\section{References}

1. Filippov, N.V., Filippova, T.I., Vinogradov, V.P., Dense, hightemperature plasma in a non-cylinderical Z-pinch compression. Nucl. Fusion Supl. 2, 577 (1962)

2. Mather, J.W., Investigation of the high energy acceleration mode in coaxial gun. Phys. Fluids S28, (1964)

3. Springham, S.V., Lee, S., Rafique, M.S., Correlated deuteron energy spectra and neutron yield for a $3 \mathrm{~kJ}$ plasma focus, Plasma Phys. Control Fusion 42, 1023 (2000)

4. Mohammadi, M.A., Sobhanian, S., Rawat, R.S., Neutron production with mixture of deuterium and krypton in Sahand Filippov type plasma focus facility. Phys. Lett. A 375, 3002 (2011)

5. Mohammadi, M.A., Verma, R., Sobhanian, S., Wong, C.S., Lee, S., Springham, S.V., Tan, T.L., Lee, P., Rawat, R.S., Neon soft X-ray emission studies from UNU-ICTP plasma focus operated with longer than optimal anode length. Plasma Sour. Sci. Tech. 16, 785 (2007)

6. Zakaullah, M., Alamgir, A., Shafiq, M., Sharif, M., Waheed, A., Scope of plasma focus with argon as a soft X-Ray source. IEEE Trans. Plasma Sci. 30, 2089 (2002)

7. Valipour, M., Mohammadi, M.A., Sobhanian, S., Rawat, R.S., Increasing of hardness of titanium using energetic nitrogen ions from Sahand as a Filippov Type plasma focus facility. J Fusion Energ. 31, 65 (2012)

8. Ghareshabani, E., Mohammadi, M.A., Measurement of the energy of nitrogen ions produced in Filippov Type plasma focus used for the nitriding of titanium. J Fusion Energ. 31, 595 (2012)
9. Patran, A., Stoenescu, D., Rawat, R.S., Springham, S.V., Tan, T.L., Tan, L.C., Rafique, M.S., Lee, P., Lee, S., A magnetic electron analyzer for plasma focus electron energy distribution studies. J. Fusion Energy 25, 57 (2006)

10. Lee, S., et al., Application of plasma focus as a source of high energy electron. Singap. J. Phys. 173, 276 (2003)

11. Kato, Y., Be, S.H., Generation of soft $\mathrm{x}$ rays using a rare gashydrogen plasma focus and its application to $\mathrm{x}$-ray lithography. Appl. Phys. Lett. 48, 686 (1986)

12. Castillo, F., Gamboa-deBuen, I., Herrera, J.J.E., Rangel, J., Villalobos, S., High contrast radiography using a small dense plasma focus. Appl. Phys. Lett. 92, 051502 (2008)

13. Ghareshabani, E., Rawat, R.S., Sobhanian, S., Verma, R., Karamat, S., Pan, Z.Y., Synthesis of nanostructured multiphase Ti(C, $\mathrm{N}) / \mathrm{aC}$ films by a plasma focus device. Nucl. Instrum. Methods Physi. Res. B 268, 2777-2784 (2010)

14. Khan, I.A., Hassan, M., Ahmad, R., Qayyum, A., Murtaza, G., Zakaullah, M., et al., Nitridation of zirconium using energetic ions from plasma focus device. Thin Solid Films 516, 8255-8263 (2008)

15. Gupta, Ruby, Srivastava, M.P., Carbon ion implantation on titanium for TiC formation using a dense plasma focus device. Plasma Sources Sci. Technol. 13, 371-374 (2004)

16. Ruby Gupta, Srivastava, M.P., Balakrishnan, V.R., Kodama, R., Peterson, M.C., Deposition of nanosized grains of ferroelectric lead zirconate titanate on thin films using dense plasma focus. J. Phys. D Appl. Phys. 37, 1091-1094 (2004)

17. Kozlov. N. P., Aleksev. V. A., Protsov. Y. S. and Rubinov. A. B., High-power ultraviolet paraterphenyl-solution laser excited by the plasma focus of a magnetoplasma compressor. JEPT Lett. 20, 331 (1974)

18. Koohestani, S., Habibi, M., Amrollahi, R., Baghdadi, R., Roomi, A., Effect of quartz and pyrex insulators length on hard-X ray signals in APF plasma focus device. J. Fusion Energy 30, 68-71 (2011)

19. Feugeas, J.N., The influence of the insulator surface in the plasma focus behavior. J. Appl. Phys. 66, 3467 (1989)

20. Rawat, R.S., Zhang, T., Phua, C.B.L., Then, J.X.Y., Chandra, K.A., Lin, X., Patran, A., Lee, P., Effect of insulator sleeve length on soft $\mathrm{x}$-ray emission from a neon-filled plasma focus device. Plasma Sour. Sci. Technol. 13, 569-575 (2004)

21. Zhang, T., Lin, X., Chandra, K.A., Tan, T.L., Springham, S.V., Patran, A., Lee, P., Lee, S., Rawat, R.S. Current sheath curvature correlation with the neon soft $\mathrm{X}$-ray emission from plasma focus device. Plasma Sources Sci. Technol. 14, 368-374 (2005)

22. Zakaullah, M., et al., Effect of insulator sleeve length on neutron emission in a plasma focus. Phys. Lett. A 137, 39 (1989)

23. Seng, Y.S., Lee, P., Rawat, R.S., Current sheath formation dynamics and structure for different insulator lengths of plasma focus device. Phys. Plasmas 21, 113508 (2014)

24. Mohammadi, M.A., Sobhanian, S., Wong, C.S., Lee, S., Lee, P., Rawat, R.S., The effect of anode shape on neon soft x-ray emissions and current sheath configuration in plasma focus device. J. Phys. D Appl. Phys. 42, 045203 (2009)

25. Yousefi, H.R., Aghamir, F.M., Masugata, K., Effect of the insulator length on Mather-type plasma focus devices. Phys. Lett. A 361, 360-363 (2007)

26. Zakaullah, M., Mrtaza, G., Ahmad, I., Beg, F.N., Beg, M.M., Shabbir, M., Comparative study of low energy Mather-type plasma focus devices. Plasma Sour. Sci. Technol. 4, 117-124 (1995)

27. Kies, W., Power limits for dynamical pinch discharges. Plasma Phys. Controll Fusion 28, 1645-1657 (1986)

28. Serban, A., Anode geometry and focus characteristics. PhD thesis, Nanyang Technological University (1995) 